Washington University School of Medicine Digital Commons@Becker

Open Access Publications

2021

\title{
Diffusion histology imaging differentiates distinct pediatric brain tumor histology
}

Zezhong Ye

Washington University School of Medicine in St. Louis

Komal Srinivasa

Washington University School of Medicine in St. Louis

Ashely Meyer

Washington University School of Medicine in St. Louis

Peng Sun

Washington University School of Medicine in St. Louis

Joshua Lin

Washington University School of Medicine in St. Louis

See next page for additional authors

Follow this and additional works at: https://digitalcommons.wustl.edu/open_access_pubs

Please let us know how this document benefits you.

\section{Recommended Citation}

Ye, Zezhong; Srinivasa, Komal; Meyer, Ashely; Sun, Peng; Lin, Joshua; Viox, Jeffrey D.; Song, Chunyu; Wu, Anthony T.; Song, Sheng-Kwei; Dahiya, Sonika; and Rubin, Joshua B., "Diffusion histology imaging differentiates distinct pediatric brain tumor histology." Scientific Reports. 11, 1. 4749 (2021).

https://digitalcommons.wustl.edu/open_access_pubs/10139

This Open Access Publication is brought to you for free and open access by Digital Commons@Becker. It has been accepted for inclusion in Open Access Publications by an authorized administrator of Digital Commons@Becker. For more information, please contact vanam@wustl.edu. 


\section{Authors}

Zezhong Ye, Komal Srinivasa, Ashely Meyer, Peng Sun, Joshua Lin, Jeffrey D. Viox, Chunyu Song, Anthony T. Wu, Sheng-Kwei Song, Sonika Dahiya, and Joshua B. Rubin 


\title{
scientific reports
}

Check for updates

\section{Diffusion histology imaging differentiates distinct pediatric brain tumor histology}

\author{
Zezhong Ye ${ }^{1,8}$, Komal Srinivasa ${ }^{2,8}$, Ashely Meyer ${ }^{3}$, Peng Sun ${ }^{1}$, Joshua Lin ${ }^{1,6}$, \\ Jeffrey D. Viox ${ }^{1,7}$, Chunyu Song ${ }^{4}$, Anthony T. Wu ${ }^{4}$, Sheng-Kwei Song ${ }^{1,4}$, Sonika Dahiya ${ }^{2 \bowtie} \&$ \\ Joshua B. Rubin ${ }^{3,5 \bowtie}$
}

High-grade pediatric brain tumors exhibit the highest cancer mortality rates in children. While conventional MRI has been widely adopted for examining pediatric high-grade brain tumors clinically, accurate neuroimaging detection and differentiation of tumor histopathology for improved diagnosis, surgical planning, and treatment evaluation, remains an unmet need in their clinical management. We employed a novel Diffusion Histology Imaging (DHI) approach employing diffusion basis spectrum imaging (DBSI) derived metrics as the input classifiers for deep neural network analysis. DHI aims to detect, differentiate, and quantify heterogeneous areas in pediatric high-grade brain tumors, which include normal white matter (WM), densely cellular tumor, less densely cellular tumor, infiltrating edge, necrosis, and hemorrhage. Distinct diffusion metric combination would thus indicate the unique distributions of each distinct tumor histology features. DHI, by incorporating DBSI metrics and the deep neural network algorithm, classified pediatric tumor histology with an overall accuracy of $\mathbf{8 5 . 8 \%}$. Receiver operating analysis (ROC) analysis suggested DHI's great capability in distinguishing individual tumor histology with AUC values $(95 \% \mathrm{Cl})$ of $0.984(0.982-0.986), 0.960(0.956-0.963)$, $0.991(0.990-0.993), 0.950(0.944-0.956), 0.977(0.973-0.981)$ and $0.976(0.972-0.979)$ for normal WM, densely cellular tumor, less densely cellular tumor, infiltrating edge, necrosis and hemorrhage, respectively. Our results suggest that DBSI-DNN, or DHI, accurately characterized and classified multiple tumor histologic features in pediatric high-grade brain tumors. If these results could be further validated in patients, the novel DHI might emerge as a favorable alternative to the current neuroimaging techniques to better guide biopsy and resection as well as monitor therapeutic response in patients with high-grade brain tumors.

Pediatric brain tumors are the second most common childhood malignancy and the most common solid tumor in children ${ }^{1}$. Pediatric brain cancer has unfortunately surpassed leukemia to become the most common cause of childhood cancer death in the US ${ }^{2}$. It is estimated that 2940 new cases of childhood (0-14 age group) and adolescent (15-19 age group) primary malignant and non-malignant central nervous system (CNS) tumors will be diagnosed in the United States in $2020^{3}$.

In the past two decades, the advent of neuroimaging technologies has enabled clinicians to detect tumor recurrence or dissemination with improved certainty ${ }^{4}$. However, these discoveries were developed largely for adult brain tumor patients, which are most often biologically distinct from those that occur in children, which exhibit unique genomic and imaging characteristics ${ }^{5}$. Evaluating pediatric brain tumors is often a diagnostic challenge due to their diverse tumor pathologies, nonspecific or overlapping imaging findings, susceptibility artifacts from intratumoral calcification or hemorrhage, and motion artifacts in young children ${ }^{6}$. Conventional MRI-based diagnoses also fail to offer adequate information regarding the specific tumor type, tumor grade, tumor viability,

${ }^{1}$ Department of Radiology, Washington University School of Medicine, Room 3221, 4525 Scott Ave., St. Louis, MO 63110, USA. 'Department of Pathology and Immunology, Washington University School of Medicine, 660 S. Euclid Avenue, St. Louis, MO 63110, USA. ${ }^{3}$ Department of Pediatrics, St. Louis Children's Hospital, Washington University School of Medicine, 660 S. Euclid Avenue, St. Louis, MO 63110, USA. "'Department of Biomedical Engineering, Washington University, St. Louis, MO 63130, USA. ${ }^{5}$ Department of Neuroscience, Washington University School of Medicine, St. Louis, MO 63110, USA. ${ }^{6}$ Present address: Keck School of Medicine, University of Southern California, Los Angeles, CA 9003389, USA. ${ }^{7}$ Present address: School of Medicine, University of Missouri - Kansas City, Kansas City, MO 64110, USA. ${ }^{8}$ These authors contributed equally: Zezhong Ye and Komal Srinivasa. ${ }^{\circledR}$ email: ssong@wustl.edu; sdahiya@wustl.edu; rubin_j@wustl.edu 
and treatment response of lesions. Although advanced MRI techniques like diffusion tensor imaging (DTI), perfusion MRI, MR spectroscopy (MRS), and susceptibility-weighted imaging (SWI) are incorporated into clinical MRI protocols, they still fall short ${ }^{6-8}$. The widely used multiparametric magnetic resonance imaging (mpMRI) approach fails to accurately reflect tumor histopathology such as tumor cellular density, necrosis, hemorrhage, or infiltrative edges. As prior studies documented the histological and radiological tumor heterogeneity coexisting within high grade tumor lesions, it is imperative to develop a technique capable of discerning the varied appearance of these lesions non-invasively'.

We have previously developed diffusion basis spectrum imaging (DBSI) ${ }^{10}$ demonstrating its ability to quantitatively characterize pathologies in multiple central nervous system diseases, including, multiple sclerosis ${ }^{11-14}$, spinal cord injury ${ }^{15}$, and epilepsy ${ }^{16}$. More recently, we developed a novel diffusion histology imaging (DHI) approach that incorporates DBSI-derived diffusion metrics as the input for a deep neural network (DNN) algorithm to detect and differentiate underlying pathologies in pediatric high-grade brain tumors.

\section{Materials and methods}

Study design. This study has been approved by the institutional review board of Washington University School of Medicine. Informed consent was obtained from all subjects' parents and/or legal guardians for the use of samples in this study. All methods were carried out in accordance with relevant guidelines and regulations. Nine post-mortem pediatric brain tumor specimens that were part of the Washington University Legacy Project were included for the study. Among these nine pediatric patients, four were male and five were female.

Postmortem brain specimen. The autopsy was performed within $24 \mathrm{~h}$ of the death of the patients to prevent the deterioration of the tissue. The brain specimen was immediately fixed in formalin right after the autopsy (Fig. 1b). The tissue blocks were obtained from the brain specimen no sooner than seven days after the formalin fixation (Fig. 1c). A total of 45 samples were resected from tumor, tumor interface with normal adjacent brain, areas of hemorrhage and necrosis, as well as normal brain tissue (Fig. 1c). The average size of the specimens was $21 \mathrm{~mm} \pm 4 \mathrm{~mm}$.

Ex vivo MRI of brain specimen. Brain tumor specimens were submersed in formalin for ex vivo imaging to keep tissue from dehydration during study. The specimens were examined using a 4.7-T Agilent/Varian MR scanner (Agilent Technologies, Santa Clara, CA) and a custom-built circular surface coil (3.5-cm diameter). A multi-echo spin-echo diffusion weighted sequence using 99 diffusion-encoding directions with maximum b-values $=3000 \mathrm{~s} / \mathrm{mm}^{2}$ was employed to acquire diffusion-weighted images. The imaging parameters were as follows: repetition time $(\mathrm{TR})=1500 \mathrm{~ms}$, echo time $(\mathrm{TE})=40 \mathrm{~ms}$, time between application of gradient pulse $20 \mathrm{~ms}$, diffusion gradient on time $8 \mathrm{~ms}$, slice thickness $0.5 \mathrm{~mm}$, field-of-view (FOV) $32 \times 32 \mathrm{~mm}^{2}$, data matrix $128 \times 128$, number of average 1 , in-plane resolution $0.25 \times 0.25 \mathrm{~mm}^{2}$. T2W images were acquired with a multislice spin echo sequence with $\mathrm{TR}=4000 \mathrm{~ms}$, echo time $\mathrm{TE}=80 \mathrm{~ms}$, FOV $32 \times 32 \mathrm{~mm}^{2}$, data matrix $128 \times 128$. T1W images were acquired with a gradient echo sequence with TR $=80 \mathrm{~ms}, \mathrm{TE}=10 \mathrm{~ms}, \mathrm{FOV} 32 \times 32 \mathrm{~mm}^{2}$, data matrix $128 \times 128,8$ averages.

DBSI analysis of brain tumor. DBSI models brain tumor diffusion-weighted MRI signals as a linear combination of discrete multiple anisotropic diffusion tensors and a spectrum of isotropic diffusion tensors:

$$
\frac{S_{k}}{S_{0}}=\sum_{i=1}^{N_{\text {Aniso }}} f_{i} e^{-\left|\overrightarrow{b_{k}}\right| \lambda_{\perp i}} e^{-\left|\overrightarrow{b_{k}}\right|\left(\lambda_{\| i}-\lambda_{\perp i}\right) \cos ^{2} \phi_{i k}}+\int_{a}^{b} f(D) e^{-\left|\overrightarrow{b_{k}}\right| D} d D \quad(k=1,2,3, \ldots)
$$

$\operatorname{In}^{1}, b_{k}$ is the $k$ th diffusion gradient; $S_{k} / S_{0}$ is the acquired diffusion-weighted signal at direction of $b_{k}$ normalized to non-diffusion-weighted signal; $N_{\text {Aniso }}$ is number of anisotropic tensors to be determined; $\phi_{i k}$ is the angle between diffusion gradient $\left(b_{k}\right)$ and principal direction of the ith anisotropic tensor; $\left|\overrightarrow{b_{k}}\right|$ is $b$-value of the kth diffusion gradient; $\lambda_{\| i}$ and $\lambda_{\perp i}$ are axial and radial diffusivity of the ith anisotropic tensor under the assumption of cylindrical symmetry; $f_{i}$ is signal-intensity-fraction of the $i$ th anisotropic tensor; $a, b$ are low and high diffusivity limits of isotropic diffusion spectrum; $f(D)$ is signal-intensity-fraction at isotropic diffusivity $D$.

Based on our ex vivo MRI and histological analyses of resected specimens from previous studies ${ }^{17}$, the following isotropic-diffusion profiles were established. We observed that highly restricted isotropic diffusion $\left(0 \leq \mathrm{D} \leq 0.2 \mu \mathrm{m}^{2} / \mathrm{ms}\right)$ is associated with lymphocytes; restricted-isotropic diffusion $\left(0.2<\mathrm{D} \leq 0.8 \mu \mathrm{m}^{2} / \mathrm{ms}\right)$ is associated with dense tumor cellularity; and hindered-isotropic diffusion $\left(0.8<\mathrm{D} \leq 2 \mu \mathrm{m}^{2} / \mathrm{ms}\right)$ is associated with tumor necrosis. DBSI provides a simple tensor expression for individual image voxels to visualize morphological features secondary to tumor formation, some of which are not as discretely detectable by conventional MRI. It is the sensitivity of diffusion-weighted MRI signal to the microstructural changes that allows DBSI to more precisely reflect morphological changes resulting from tumor presence or other pathologic alterations. By using this feature of DBSI as the input for deep neural network algorithms, we created DHI to recapitulate histopathologic analysis using MRI.

Histologic staining and evaluation. The formalin-fixed tissue was embedded in paraffin after scanning. The paraffin embedded tissue was then sequentially sectioned at 5- $\mu \mathrm{m}$ thickness and stained with hematoxylin and eosin (H\&E). Histology slides were digitized using NanoZoomer 2.0-HT System (Hamamatsu, Japan) with a $20 \times$ objectives for analyses. Two experienced neuropathologists (K.S. and S.D.) reviewed all the histological slides with a consensus on the selected tumor histopathologic features. Regions of normal white matter (WM), 

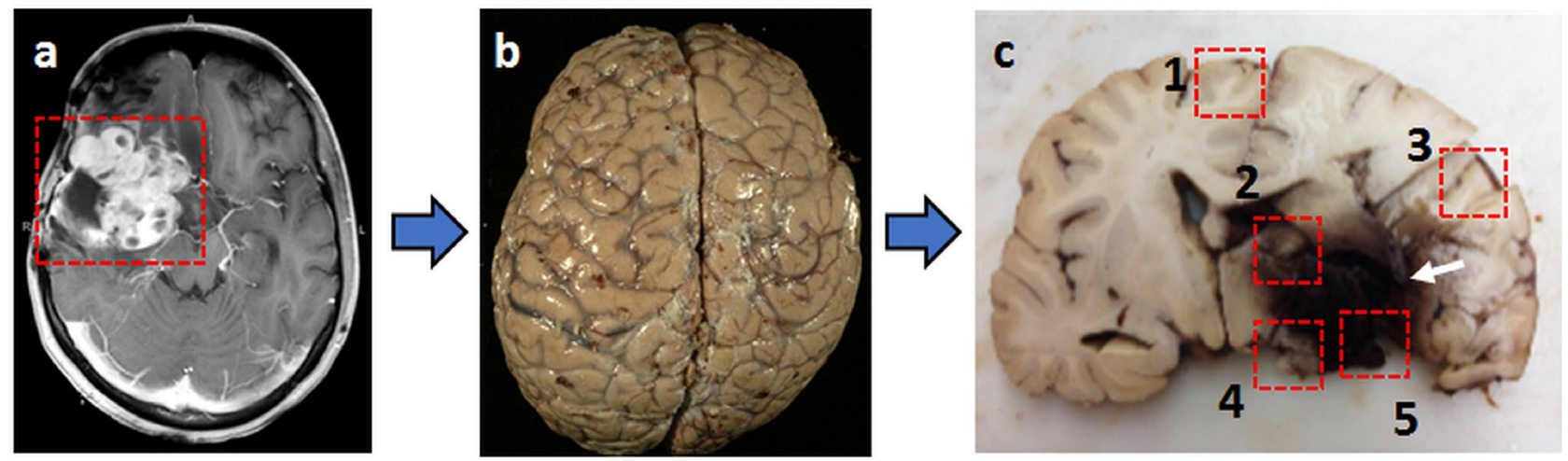

\section{d}
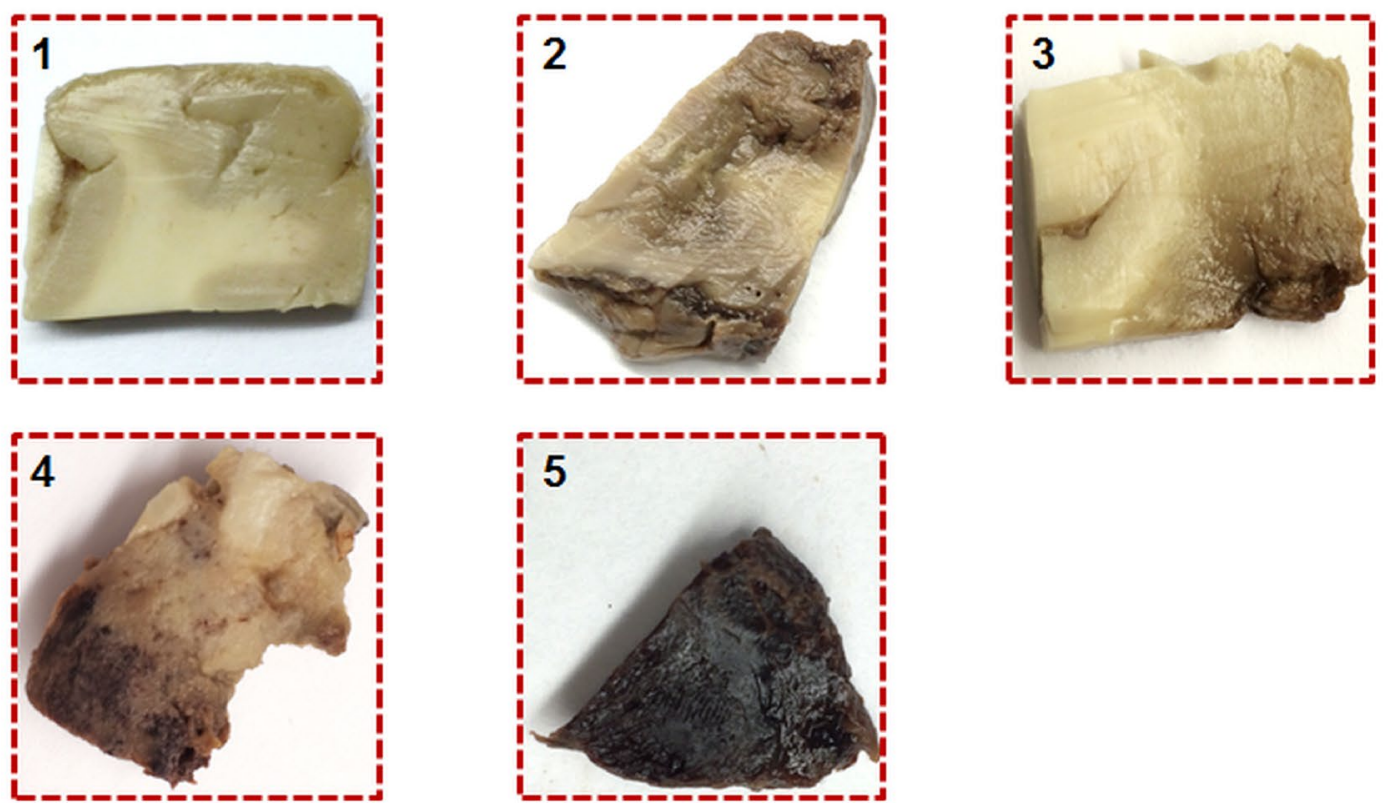

Figure 1. Illustration of brain specimen procurement from a patient with high-grade pediatric brain tumor. (a) In vivo Gd-enhanced T1-weighted image indicated a large lesion (square) with heterogeneous intensities in the right posterior region from a 16-year-old patient with embryonal neoplasm (WHO Grade IV). (b) Brain specimen was procured and immediately formalin-fixed. (c) Coronal slices revealed a large tumor with admixed hemorrhage and necrosis in the right thalamus (arrow). (d) Five tissue blocks were prepared in total i.e. from tumor (block 2, blcok 4), tumor interface with normal adjacent brain (block 3), hemorrhage and necrosis (block $5)$, as well as grossly normal brain (block 1) (c).

densely cellular tumor (DC tumor), less densely cellular tumor (LDC tumor), necrosis, tumor infiltrative edge, and hemorrhage were outlined and drawn on $\mathrm{H} \& \mathrm{E}$ images with $20 \times$ magnification.

Image processing. Voxel-wise DTI and DBSI analyses were performed by an in-house software developed using MATLAB $^{\circledast}$ (MathWorks; Natick, MA). The computation time for DTI and DBSI for each specimen is about $40 \mathrm{~min}$.

Co-registration between histology images and ex vivo MRI. The two dimensional (2D) thin plate spline (TPS) registration was performed using MIPAV (Version 10.0.0, NIH; https://mipav.cit.nih.gov/index .php) as described in our previous study ${ }^{17}$ to co-register the histology images with MR images. To achieve successful co-registration, we first ensured the plane of histology section of the brain tumor specimens matched closely with the slice plane of the corresponding T2-weighted (T2W) images. For the co-registration preprocessing, the RGB format of H\&E images with histology annotations were converted to grayscale format to match with T2W images (Fig. 2) using the Pillow package in Python 3.6.8 (https://pillow.readthedocs.io/en/3.1.x/index .html\#). Afterwards, eighteen pairs of landmarks along the perimeter of each specimen were manually placed on both H\&E images and T2W images (inherently co-registered with DTI and DBSI maps) to compute the transformation matrix for matching H\&E images with MRI (Fig. 2). Through successful image co-registration, 

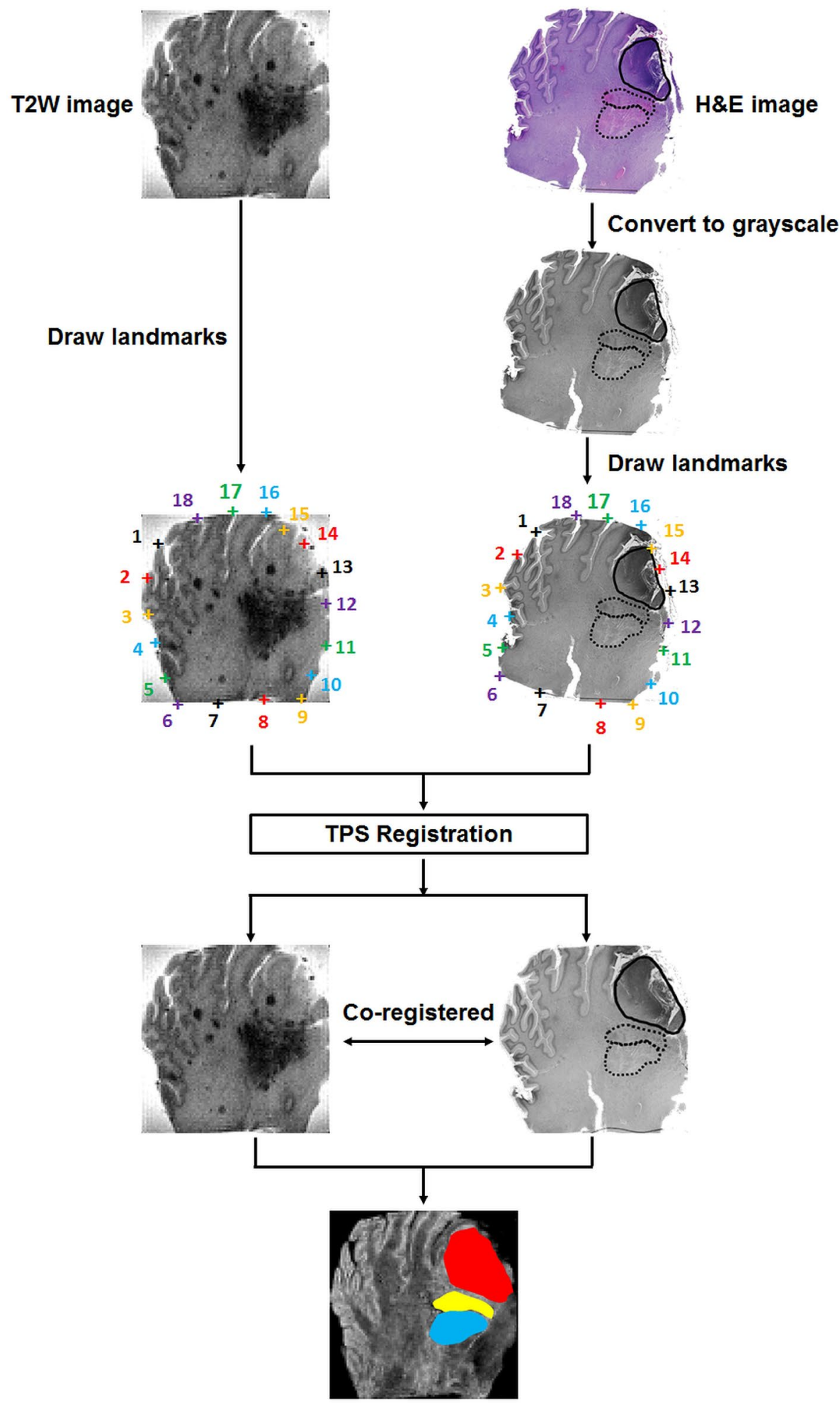

Histologically-defined ROI on DBSI

Figure 2. Co-registration between histology and MRI. Raw RGB H\&E images were first converted to grayscale images for enhanced coregistration. Eighteen pairs of landmarks along the perimeter of the brain specimens were manually placed on the MR image and grayscale H\&E image. The transformation matrix of the twodimensional thin plate spline (TPS) registration was computed in MIPAV (version 10.0.0) and applied to warp the H\&E image to the orientation of the MR image. After co-registration, the pathologist defined tumor histology regions on H\&E images. These were then successfully transferred to the corresponding MR image. 
the regions of interest (ROI) with pathologist-identified tumor histology on the H\&E images can be transferred to DBSI for further analysis (Fig. 2).

Deep neural network (DNN) model development and optimization. Our complete dataset consisted of 99,388 imaging voxels from 45 specimens obtained from 9 patients. The collected voxels were split into training, validation, and test datasets with an 8:1:1 ratio, respectively. Imaging voxels from test datasets were separated and distinct from the ones that were used in the training and validation steps. Validation set was employed to fine tune the model hyper-parameters. To balance data from groups of different tumor histologic components, a synthetic minority oversampling technique (SMOTE) ${ }^{18}$ was applied to over-sample the minority group by introducing synthetic feature samples. This data balancing approach has been demonstrated to be beneficial for avoiding over-fitting and improving model generalization ${ }^{18,19}$. Data balancing were only applied to the training dataset, while the validation and test dataset was kept unchanged. The diffusion metrics assessed with our DNN modeling included 10 diffusion metrics provided from DBSI. Specifically, DBSI metrics include mean apparent diffusion coefficient (ADC), mean FA, fiber fraction, fiber fractional anisotropy (FA), fiber axial diffusivity (AD), fiber radial diffusivity (RD), restricted isotropic diffusion fraction (restricted fraction), restricted isotropic diffusivity, hindered isotropic diffusion fraction (hindered fraction), hindered isotropic diffusivity, free isotropic diffusion fraction (free fraction), free isotropic diffusivity.

A supervised deep neural network (DNN) was adopted to detect and classify tumor histologic components by referencing the H\&E findings. The DNN model was developed using TensorFlow 2.0 framework in Python $3.6 .8^{20}$. In general, the DNN model was equipped with ten fully connected hidden layers. Batch normalization layer with a mini-batch size of 200 was used before feeding data to the next hidden layer to improve model optimization and prevent overfitting. Exponential linear units ${ }^{21}$ were adopted to activate specific functions in each hidden layer. The final layer was a fully connected softmax layer that generated a likelihood distribution of six output classes. We used Adam optimizer with the default parameters of $\beta_{1}=0.9, \beta_{2}=0.999$ and mini-batch size of 200. The learning rate was manually tuned to achieve the fastest convergence. We chose cross-entropy as the loss function and trained the model to minimize the error rate on the validation dataset. Overall, hyperparameters for the DNN architecture and optimization algorithm were chosen through a combination of grid search and manual tuning.

Statistical analysis. Statistical differences in diffusion metrics between the tumor histology groups were evaluated by the Mann-Whitney $U$ test. The results were presented as mean \pm standard deviation. A $p<0.05$ was considered statistically significant. In multi-class classification, confusion matrices were calculated and used to illustrate the specific examples of tumor histologic components where the model prediction agrees with the pathologists' diagnoses. We also used one-versus-rest strategy to perform receiver operating characteristics (ROC) analysis. Area under curve (AUC) was calculated to assess model discrimination of each tumor histological component. Sensitivity and specificity values were calculated using Youden Index ${ }^{22}$. The precision-recall curve and $\mathrm{F}_{1}$-scores were also calculated to provides complementary information to the ROC curves. $\mathrm{F}_{1}$-score (ranges from 0 to 1 ) favors models that maximize both precision and recall simultaneously, which is especially helpful to address the insensitivity of AUC on class imbalance. The $95 \%$ confidence interval values were calculated using the percentile bootstrap method with 10,000 independent experiments ${ }^{23}$. All the statistical metrics and curves were calculated using the $\mathrm{SciPy}^{24}$ and Scikit-learn ${ }^{25}$ packages with Python version 3.6.

\section{Results}

A brief description of tumor specimens examined. Postmortem brain tumor specimens examined were from 9 pediatric brain tumor patients, aged from 4 to 17 years old at the time of initial diagnosis. The mean age was $10.8 \pm 3.7$ years old. The patients' age at autopsy ranged from 7 to 18 , with a mean of $13.1 \pm 3.7$ years. Four tumors were located in the brainstem, two in the thalami, one in the right cerebral cortex, and one at the cerebellopontine angle. These were confirmed to be diffuse midline gliomas with H3K27M mutation by immunohistochemistry $(n=4)$, glioblastoma $(n=3)$, and embryonal tumor with multilayered rosettes with LIN28A protein overexpression (medulloepithelioma phenotype, NEC; $n=1$ ). One patient with neurofibromatosis $1(\mathrm{NF} 1)$ had three different tumors at three distinct time points; these were an optic pathway glioma (pilocytic astrocytoma), a diffuse astrocytoma, WHO grade II involving the right parieto-temporal lobe, and a CNS embryonal tumor involving the right temporal lobe. All details are summarized in Table 1.

Relating MRI metrics to tumor pathologies. Figures 1 and 3 show a representative case from a 16-yearold brain tumor patient with embryonal neoplasm (WHO Grade IV). Clinical gadolinium (Gd)-enhanced T1-weighted imaging of this patient several weeks prior to death revealed a new lesion in the right temporal lobe of the brain (Fig. 1a, square). At autopsy, the brain was removed and immediately suspended in formalin for fixation (Fig. 1b). Coronal slices exhibited a large hemorrhagic and necrotic tumor mass with its epicenter in the right thalamus (Fig. 1c). Tissue blocks were obtained from this region (Fig. 1d) for ex vivo imaging (Fig. 2). Of note, this patient had two other known tumors, one in his optic pathway (WHO grade I) and another diffuse astrocytoma (WHO grade II) in right posterior temporo-parietal lobe. The boundaries of latter were however relatively indistinct from the high-grade hemorrhagic and necrotic embryonal neoplasm (WHO grade IV) by gross examination alone.

A densely cellular tumor region and white matter (WM) were indistinguishable in both T1-weighted image (T1WI) and T2-weighted image (T2WI) (Fig. 3). Hemorrhage appeared hypointense compared to other regions in T1WI and T2WI (Fig. 3). White matter and the hemorrhagic region appeared hyperintense in diffusion-weighted 


\begin{tabular}{|c|c|c|c|c|c|c|}
\hline Patient ID & Age at diagnosis & Age at post-mortem & Gender & Location & Histologic diagnosis & Molecular alterations \\
\hline WU-1 & 9 & 9 & $\mathrm{~F}$ & Thalamus & $\begin{array}{l}\text { Diffuse midline glioma, WHO } \\
\text { grade IV }\end{array}$ & $\begin{array}{l}\text { H3K } 27 \mathrm{M} \text { mutant (by immunohis- } \\
\text { tochemistry }\end{array}$ \\
\hline WU-2 & 11 & 14 & M & Left temporal lobe & $\begin{array}{l}\text { Glioblastoma, IDH wildtype, } \\
\text { WHO grade IV }\end{array}$ & $\begin{array}{l}\text { Tumor progressed from IDH } \\
\text { wildtype anaplastic astrocytoma. } \\
\text { Next generation sequencing } \\
\text { showed CREBBP G1479 alteration }\end{array}$ \\
\hline WU-3 & 11 & 12 & $\mathrm{~F}$ & Right parietal-occipital lobe & $\begin{array}{l}\text { Diffuse midline glioma, WHO } \\
\text { grade IV }\end{array}$ & $\begin{array}{l}\text { H3K 27M (by immunohistochem- } \\
\text { istry }\end{array}$ \\
\hline \multirow{3}{*}{ WU-4 } & \multirow{3}{*}{7} & \multirow{3}{*}{16} & \multirow{3}{*}{ M } & 1. Right temporal lobe & $\begin{array}{l}\text { CNS embryonal tumor with } \\
\text { anaplastic features, WHO grade } \\
\text { IV (2013) }\end{array}$ & $\begin{array}{l}\text { Background of NF1 with three } \\
\text { tumors at different time points }\end{array}$ \\
\hline & & & & $\begin{array}{l}\text { 2. Right posterior temporoparietal } \\
\text { lobe }\end{array}$ & $\begin{array}{l}\text { Diffuse astrocytoma, WHO grade } \\
\text { II (2006) }\end{array}$ & $\begin{array}{l}18 \text { non-synonymous variants } \\
\text { were identified by next generation } \\
\text { sequencing TP53, p.R213Dfs }{ }^{\star} 34, \\
\text { TP53 and p.T211I } \\
\end{array}$ \\
\hline & & & & 3. Optic pathway & $\begin{array}{l}\text { Pilocytic astrocytoma, WHO grade } \\
\text { I (not sampled until post-mortem) }\end{array}$ & $M A P 2 K 2$, p.I369V \\
\hline WU-5 & 10 & 10 & M & Pons & $\begin{array}{l}\text { Diffuse midline glioma, WHO } \\
\text { grade IV }\end{array}$ & $\begin{array}{l}\text { H3K } 27 \mathrm{M} \text { mutant (by immunohis- } \\
\text { tochemistry }\end{array}$ \\
\hline WU-6 & 13 & 14 & M & Pons & $\begin{array}{l}\text { Diffuse midline glioma, WHO } \\
\text { grade IV }\end{array}$ & $\begin{array}{l}\text { H3K } 27 \mathrm{M} \text { mutant (by immunohis- } \\
\text { tochemistry }\end{array}$ \\
\hline WU-7 & 4 & 7 & $\mathrm{~F}$ & Right cerebellopontine angle & $\begin{array}{l}\text { Embryonal tumor with multilay- } \\
\text { ered rosettes, medulloepithelioma } \\
\text { phenotype, WHO grade IV, NEC }\end{array}$ & $\begin{array}{l}\text { FISH could not demonstrate } \\
\text { C19MC alteration but multifocal } \\
\text { LIN28A protein expression was } \\
\text { seen by immunohistochemistry }\end{array}$ \\
\hline WU-8 & 17 & 18 & M & $\begin{array}{l}\text { Right cerebral hemisphere } \\
\text { (extensive involvement left side, } \\
\text { brainstem and cerebellum) }\end{array}$ & $\begin{array}{l}\text { Glioblastoma, IDH wildtype, } \\
\text { WHO grade IV }\end{array}$ & $\begin{array}{l}\text { Loss of } 10 \mathrm{q}(P T E N) \text { and monosomy } \\
10 \text { (by FISH); no EGFR amplifica- } \\
\text { tion or polysomy of chromosome } 7\end{array}$ \\
\hline WU-9 & 15 & 18 & $\mathrm{~F}$ & $\begin{array}{l}\text { Epicenter in brainstem, right } \\
\text { thalamus, right basal ganglia, and } \\
\text { cerebellum }\end{array}$ & $\begin{array}{l}\text { Glioblastoma, IDH wildtype, } \\
\text { WHO grade IV }\end{array}$ & $\begin{array}{l}\text { H3K } 27 \mathrm{M} \text { negative (by immunohis- } \\
\text { tochemistry) }\end{array}$ \\
\hline
\end{tabular}

Table 1. Patient information.

a

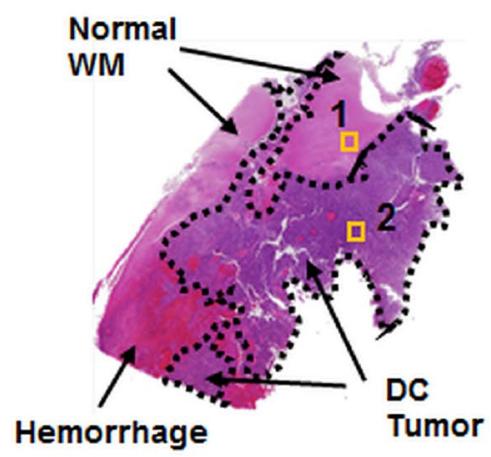

b

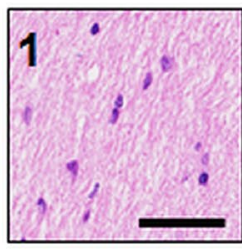

Normal WM

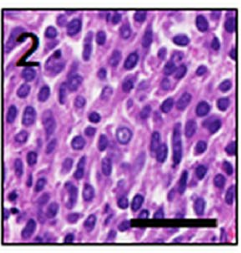

DC Tumor

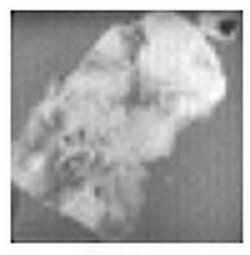

T1W

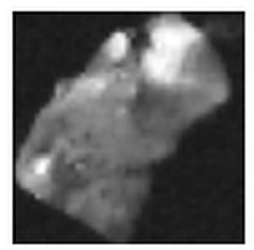

DWI
Multi-Parametric MRI

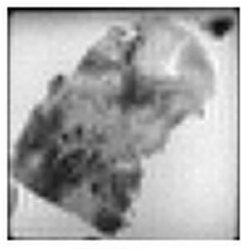

T2W

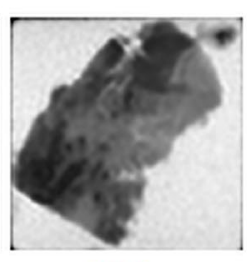

ADC

\section{DBSI}

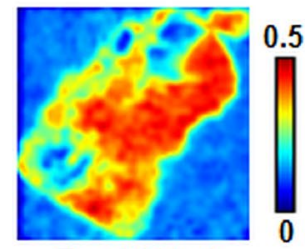

Restricted

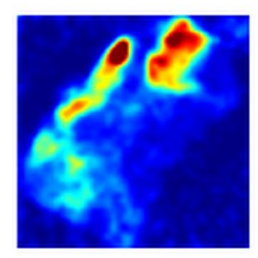

Anisotropic

Figure 3. A representative tissue block was imaged with ex vivo MRI, followed by histologic processing and evaluation. (a) H\&E image of a sectioned specimen after ex vivo MRI with regions of white matter, densely cellular tumor and hemorrhage outlined for assessing the efficacy of metrics derived by multi-parametric MRI and DBSI. The expanded region of densely cellular tumor features characteristically increased cellularity. Scale bar measures $50 \mathrm{um}$. (b) T1W and T2W MRI did not distinguish densely cellular tumor region from white matter or hemorrhage. Most strikingly, diffusely cellular tumor region exhibited lower DWI and higher ADC countering the conventional wisdom that higher tumor cellularity is associated with restricted diffusion. WM white matter, $D C$ tumor densely cellular tumor. 
image (DWI) and hypointense in ADC map comparing with the densely cellular tumor regions. Densely cellular tumor appeared to associate with high restricted fraction.

DBSI derived diffusion metrics reflected tumor pathologies. After MR images were co-registered with $\mathrm{H} \& \mathrm{E}$ images, image-voxels from segmentations of the five different tumor pathologies were subsequently obtained and plotted for group comparison (Fig. 4). The ADC of densely cellular tumor $\left(0.43 \pm 0.17 \mu \mathrm{m}^{2} / \mathrm{ms}\right)$ was $115 \%$ higher $(p<0.05)$ than then normal white matter and $42 \%$ higher $(p<0.05)$ than the infiltrative edge $\left(0.30 \pm 0.15 \mu \mathrm{m}^{2} / \mathrm{ms}\right)$. Additionally, the densely cellular tumor was $17 \%$ lower $(p<0.05)$ than the less densely cellular tumor $\left(0.52 \pm 0.26 \mu \mathrm{m}^{2} / \mathrm{ms}\right)$, and $37 \%$ lower $(p<0.05)$ than the necrosis $\left(0.68 \pm 0.37 \mu \mathrm{m}^{2} / \mathrm{ms}\right)$ (Fig. $\left.4 \mathrm{a}\right)$. DTI fractional anisotropy (FA) value of the tumor infiltrative edge was similar to that of white matter $(0.23 \pm 0.14$ vs. $0.24 \pm 0.11$ ) (Fig. 4 b). The comparison of isotropic ADC values derived by DBSI among tumor pathologies revealed a similar trend as seen in mean ADC and were consistently higher than mean ADC (Fig. 4c). For the highly restricted fraction, WM exhibited much higher values than other pathologies. Densely and less densely cellular tumors exhibited the least highly restricted fraction values among all histologic features (Fig. 4d). For the restricted fraction, densely cellular tumor $(0.35 \pm 0.10)$ showed $35 \%, 21 \%, 21 \%$ and $59 \%$ higher (all $p<0.05$ ) values than normal WM $(0.26 \pm 0.11)$, less densely cellular tumor $(0.29 \pm 0.09)$, infiltrative edges $(0.29 \pm 0.12)$ and necrosis $(0.22 \pm 0.15)$, respectively. This result correlated well with the expected cellularity decrease from densely cellular, less densely cellular tumor regions to necrotic tissue (Fig. 4e). As expected, necrosis was characterized by higher hindered fraction $(0.42 \pm 0.21)$ and free fraction values $(0.11 \pm 0.12)$ than any of the other histologic features. In the anisotropic fraction, normal WM $(0.38 \pm 0.12)$ and infiltrative edge $(0.36 \pm 0.17)$ had similar values; this anisotropic component was much higher than other histologic components.

Classifications of tumor histologic components. It was clear that DBSI is more specific to tumor pathologies than conventional MRI metrics. To alleviate overlap between DBSI-derived metrics and tumor pathologies, we sought to classify tumor pathologies using DHI, by combining DBSI-derived structural metrics with DNN algorithm. We first performed a multi-class classification of normal WM, densely cellular tumor, less densely cellular tumor, infiltrative edge, necrosis, and hemorrhage regions containing a total of 143,100 image voxels to train the DHI model after oversampling for data balancing. Representative H\&E images corresponding to one MRI voxel revealed distinct histologic features (Fig. 5a). For the independent test set $(n=9939)$, we achieved an overall accuracy of $85.8 \%$. Confusion matrix analysis indicated strong concordance between DHI predictions and the neuropathologist-identified pathological features (Fig. 5b). DHI accurately predicted normal WM, densely cellular tumor, less densely cellular tumor, infiltrative edge, necrosis, and hemorrhage, with true positive rates of $91.6 \%, 80.6 \%, 90.8 \%, 80.2 \%, 81.4 \%$ and $86.7 \%$, respectively.

To test DHI's ability to distinguish each individual tumor pathology, we adopted a one-versus-rest strategy to perform receiver operating characteristic (ROC) and precision-recall analysis (Fig. 6). The ROC curves indicated high area under curve (AUC) values in the differentiation of all six different histologic components (Fig. 6a-f). We calculated $95 \%$ confidence intervals (CI) of AUCs using the percentile bootstrap method with 10,000 iterations. The AUC values (95\% CI) were 0.984 (0.982-0.986), 0.960 (0.956-0.963), 0.991 (0.990-0.993), 0.950 (0.944-0.956), 0.977 (0.973-0.981) and $0.976(0.972-0.979)$ for normal WM, densely cellular tumor, less densely cellular tumor, infiltrative edge, necrosis and hemorrhage, respectively (Table 2). We also calculated sensitivity and specificity for each class under the Youden Index. All the sensitivity values were higher than $91 \%$ with specificity values higher than $85 \%$ (Table 2 ). We also calculated precision-recall curves and $\mathrm{F}_{1}$-scores to provide complementary information to address ROC analyses' insensitivity to class imbalance and the possible overestimation of model performance. The precision-recall curves performed inferiorly on tumor infiltration (Fig. 6d, AUC 0.747) and necrosis (Fig. 6e, AUC 0.851) when compared to other tumor histologic regions. Similarly, the $\mathrm{F}_{1}$-scores of the infiltrative edge $(0.698)$ and necrosis $(0.799)$ were worse than those of normal white matter (0.918), densely cellular tumor (0.850), less densely cellular tumor (0.911), and hemorrhage (0.848) (Table 2).

\section{Discussion}

Pediatric brain tumors are the leading cause of cancer-related death in children. Current curative approaches in management rely, in most cases, on complete surgical resection, followed by irradiation and chemotherapy ${ }^{4,26}$. Histologic assessment of tumor cellularity, infiltration and necrosis is critical in the diagnosis and grading, as well as subsequent clinical decision-making for patient management and follow-up ${ }^{27}$. The current clinical gold standard, i.e. histologic examination, requires stereotactic biopsy or surgical resection ${ }^{28}$, which carries potential risks including infections, seizures, stroke, coma, as well as brain swelling or bleeding ${ }^{29}$. Sometimes inconclusive pathological findings result from inadequate sampling, necessitating repeat biopsy, with all its attendant risks ${ }^{30}$. A noninvasive neuroimaging approach to facilitate diagnosis or guide surgical planning will ensure better treatment response assessment, ultimately improving patient care ${ }^{31}$.

While MRI remains the most common clinical imaging technique for evaluating CNS tumors ${ }^{5}$, conventional MRI sequences such as T1WI and T2WI correlated poorly with pathologies of high-grade brain tumors. For example, hyperintense regions in T2W and FLAIR images surrounding the Gadolinium (Gd)-enhancing lesion cannot distinguish between infiltrative tumor, vasogenic edema, or immune cell ${ }^{32}$. Gd-enhancement in T1WI also could occur due to either tumor progression or radiation necrosis ${ }^{33}$. Furthermore, conventional T1W and T2W image contrasts vary from scan to scan and are not quantitative, as they depend not only on the MR characteristics of brain tissue, but also on the scanner models, magnet strength, and pulse sequences.

To address the limitations of conventional MRI bridging the gap between histology and MRI for pediatric brain tumor diagnoses, we developed a novel image processing technique, i.e., DHI, taking advantage of previously developed DBSI and DNN algorithm. DBSI provides a simple tensor expression to visualize morphological 
a

a

C
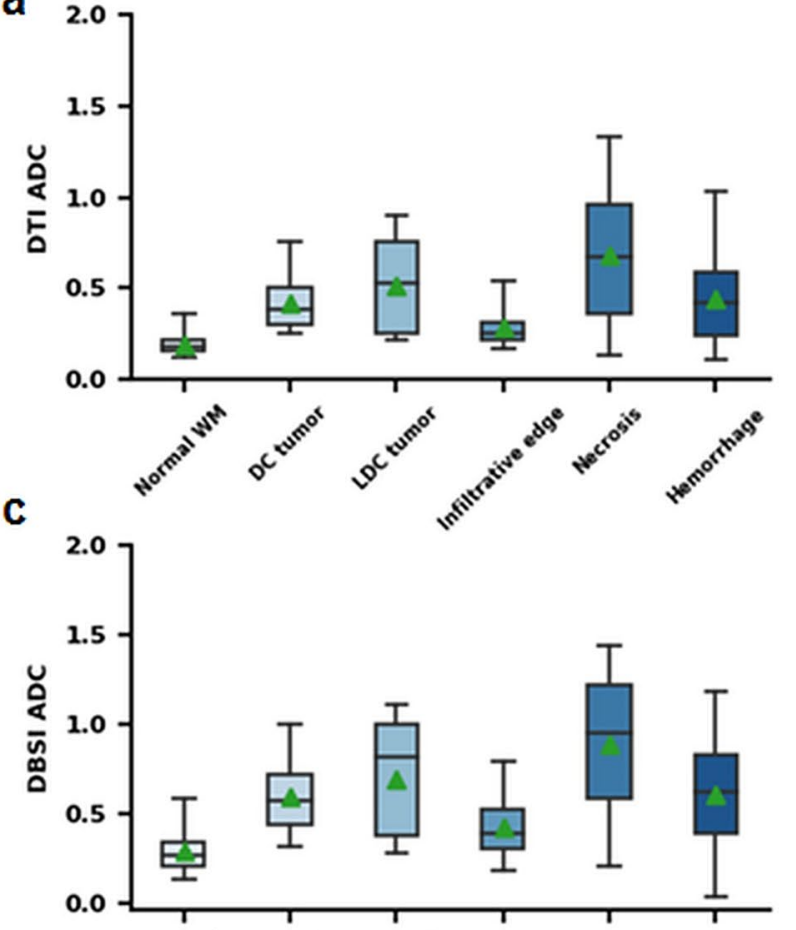

e
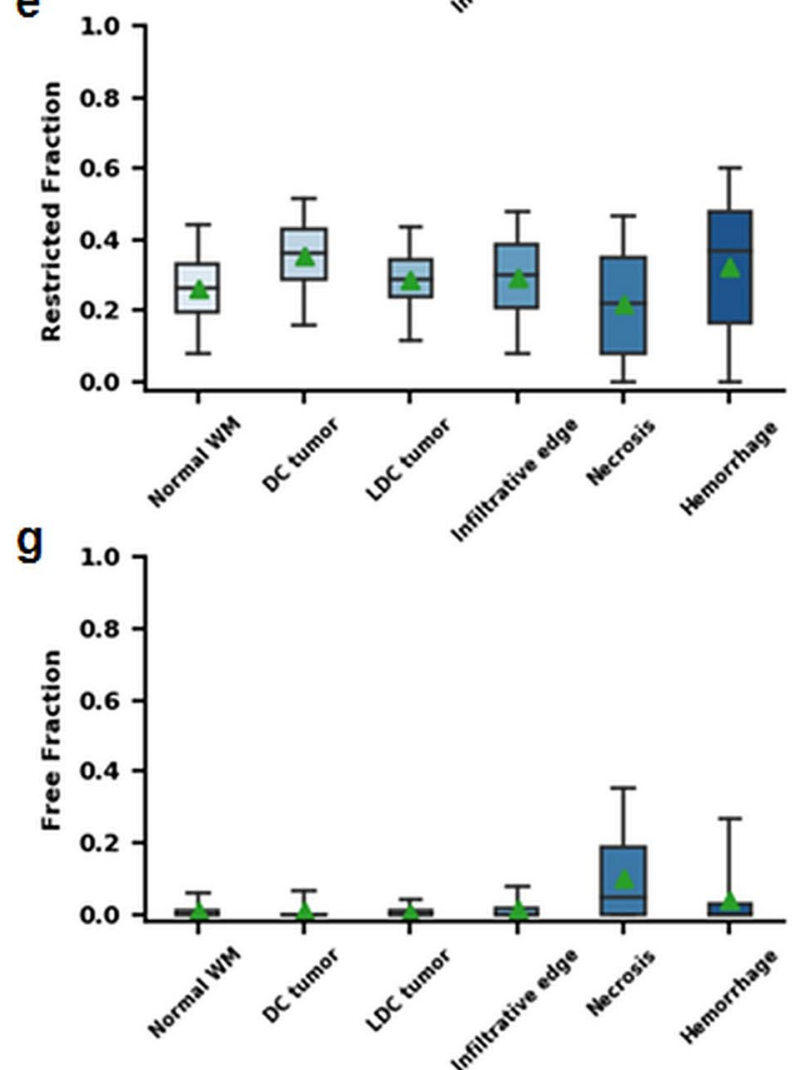

b
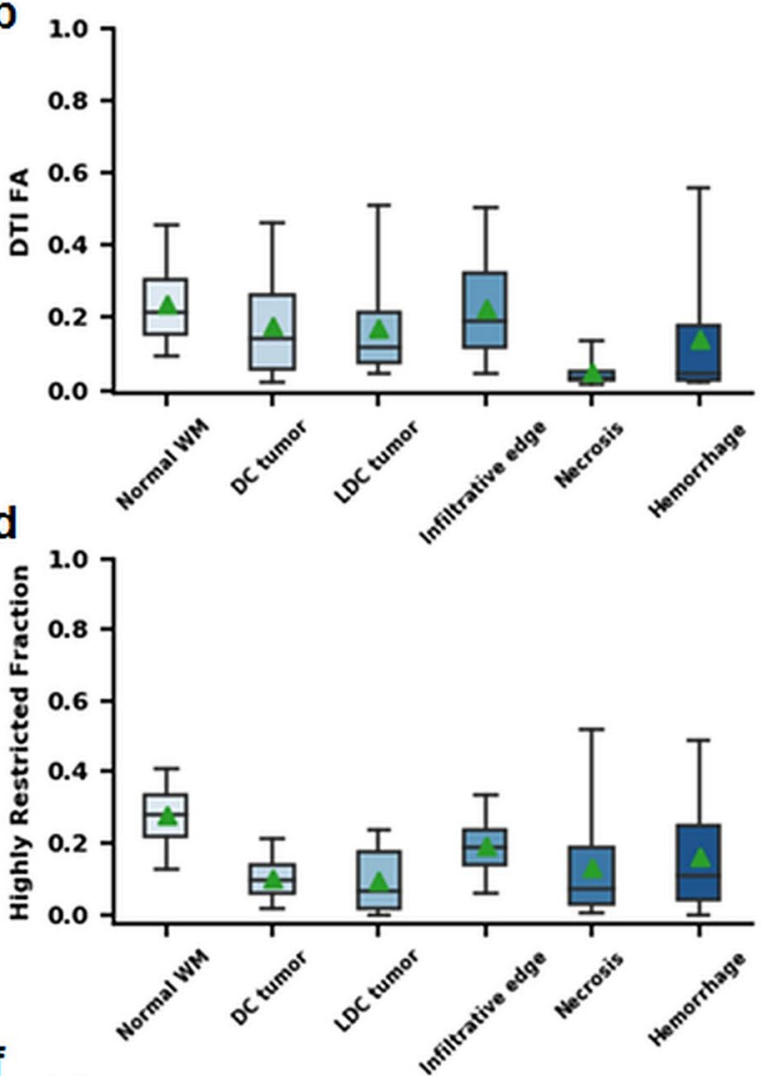

f

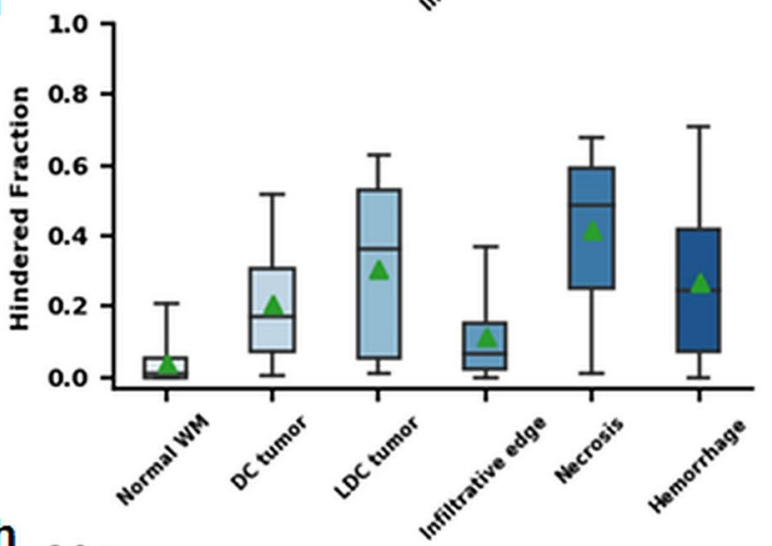

h

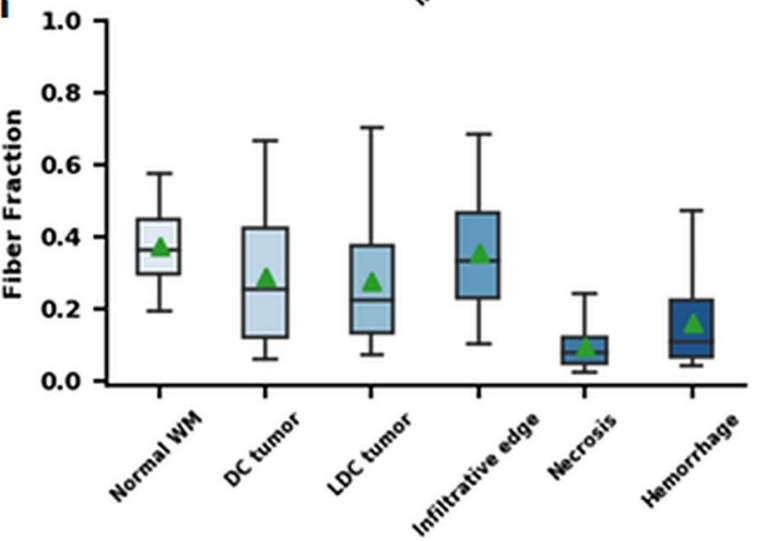

Figure 4. Group analysis on different tumor histologic components on representative diffusion metrics including (a) ADC, (b) DTI FA, (c) DBSI isotropic ADC, (d) highly restricted fraction, (e) restricted fraction, (f) hindered fraction, (g) free fraction, and (h) fiber fraction. Particularly, normal WM and the infiltrative edge showed higher fiber fraction and DTI-FA than the other tumor histologies. DC tumor and LDC tumor showed higher restricted fraction values than other histologies. Necrosis showed higher ADC, hindered fraction and free fraction values as well as lower restricted fraction, fiber fraction and DTI-FA compared to the other histologies. These findings were collectively consistent with DBSI's modelling for malignant brain tumor. ADC, $\mu \mathrm{m}^{2} / \mathrm{ms}$. Normal WM normal white matter, DC tumor densely cellular tumor, LDC tumor less densely cellular tumor. 
a

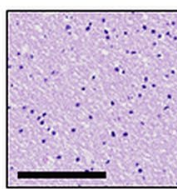

Normal WM

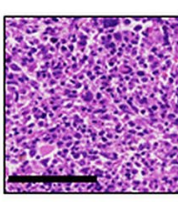

DC tumor

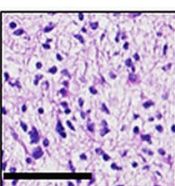

LDC tumor

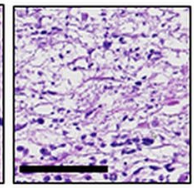

Infiltrative edge

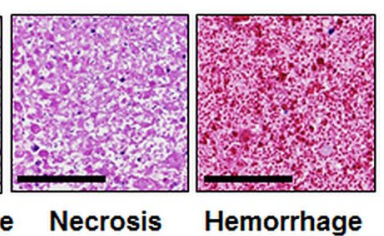

b

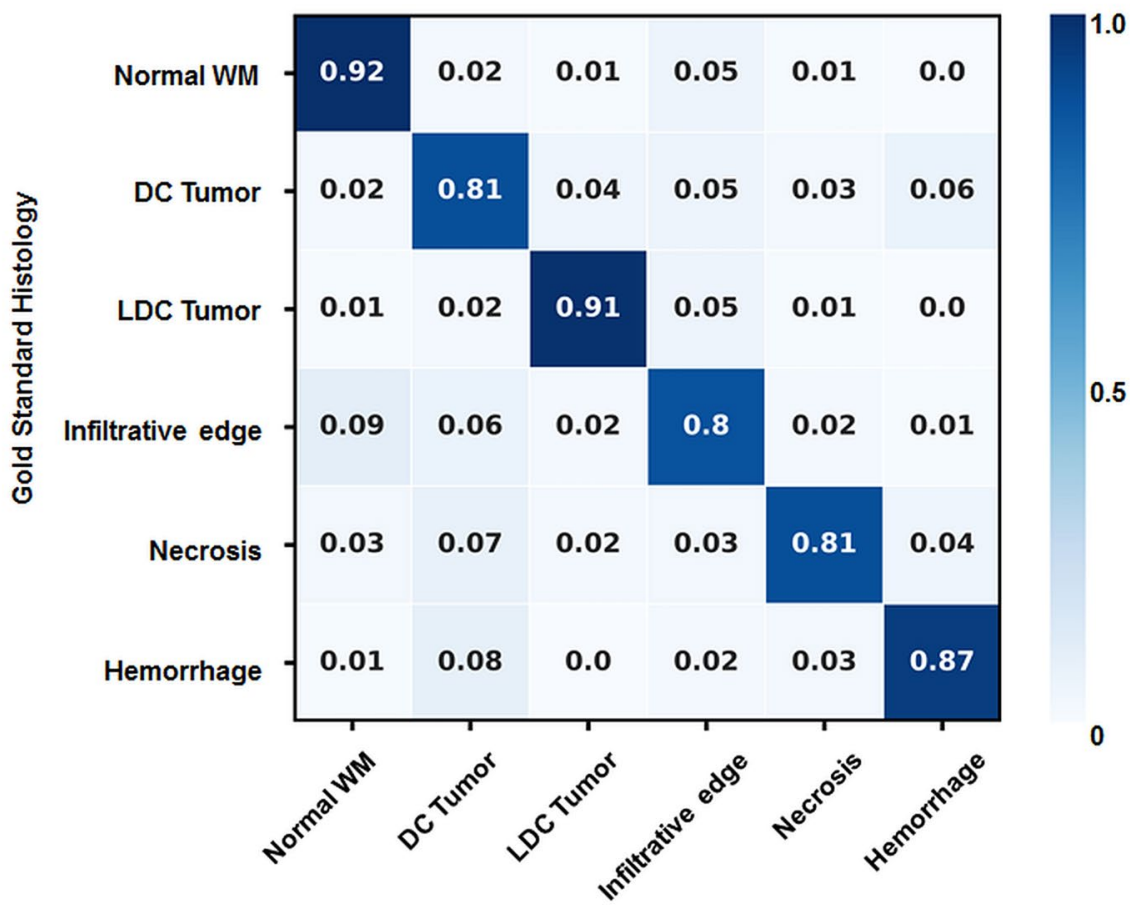

Model Predicted Histology

Figure 5. (a) Representative H\&E images of normal white matter, densely cellular tumor, less densely cellular tumor, infiltrative edge, necrosis and hemorrhage, respectively. (b) Independent test dataset confusion matrix for the predictions of DHI versus gold standard, i.e. histologic examination $(n=9939)$. Rows contain tumor histologic classifications identified by a neuropathologist, and columns represent tumor histologic classifications as predicted by DHI. Scale bar measures $100 \mu \mathrm{m}$. Normal WM normal white matter, DC tumor densely cellular tumor, LDC tumor less densely cellular tumor.

features resulting from both tumor and non-tumor elements of the brain that are indistinguishable by conventional MRI. In our previous studies, we demonstrated how DBSI-derived restricted fraction positively correlated with adult GBM tumor cellularity identified by H\&E staining ${ }^{17}$. In this study, we demonstrated that the hyperintense restricted fraction regions also accurately identify densely cellular tumor areas (Fig. 2). Group analysis across multiple samples with various tumor types also indicated densely cellular tumor had higher restricted fraction values than either normal WM, less densely cellular tumor, infiltrative edges or necrosis. From the areas of necrosis, infiltrating edge, less densely cellular tumor, and densely cellular tumor, we observed a trend towards a gradual increase in restricted fraction values across these four types of histological areas. Thus, the restricted fraction could serve as an appropriate biomarker to assess tumor cellularity in high-grade pediatric brain tumors.

In addition, necrosis exhibited higher values in hindered diffusion fraction and free diffusion fraction than all other histologic components, indicating that these two diffusion metrics are strongly associated with tumor necrosis. Furthermore, our results showed comparable ADC, FA, restricted fraction, and fiber fraction values between the infiltrative edge and normal WM, suggesting that these diffusion metrics lack adequate specificity to distinguish between infiltrating tumor cellularity and white matter. Note that infiltrative edges showed higher isotropic $\mathrm{ADC}$ and hindered fraction values than do normal WM, potentially pointing to how tumor infiltration displaces normal parenchyma ${ }^{34}$, destructs of white matter tracts ${ }^{35}$, and/or forming vasogenic edema to disrupt blood brain barrier ${ }^{36}$.

In this study, we demonstrated that DHI, i.e., DBSI-derived metrics as the input classifiers for DNN, differentiates 6 major types of tumor pathologies with an overall accuracy of 85.8\%. In detecting and distinguishing individual tumor histology, ROC analysis of DHI models calculated the AUC, sensitivity, and specificity values of all selected tumor pathologies to be higher than $0.94,88 \%$ and $86 \%$, respectively. In the precision-recall analysis, 
a

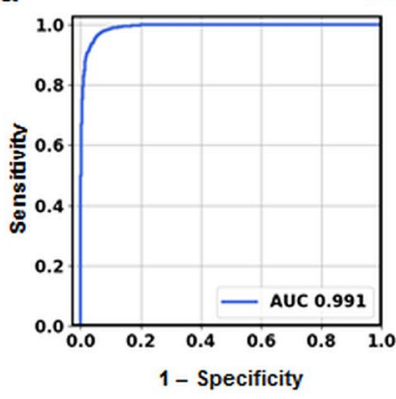

Normal WM

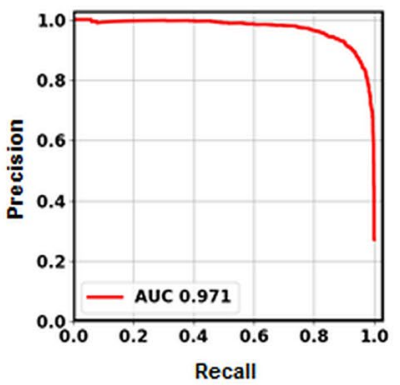

b

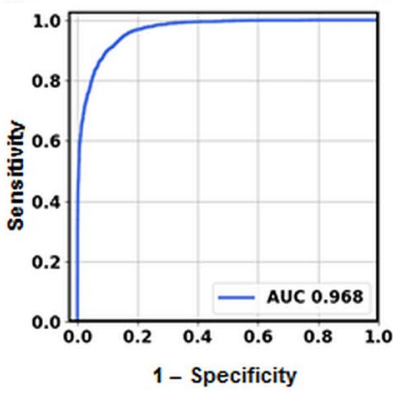

DC tumor

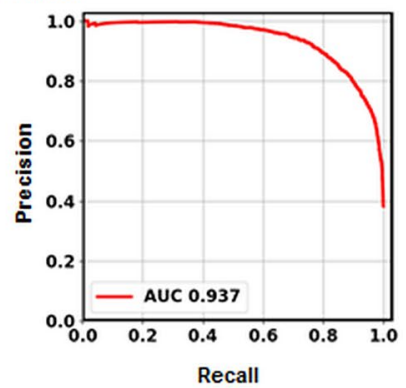

C

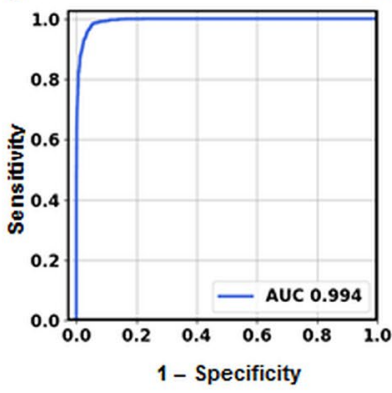

LDC tumor

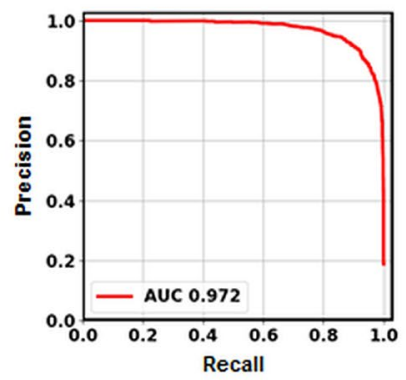

d

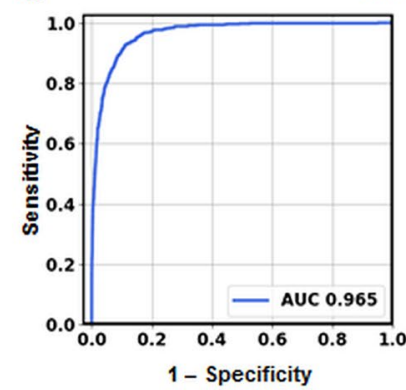

Infiltrative edge

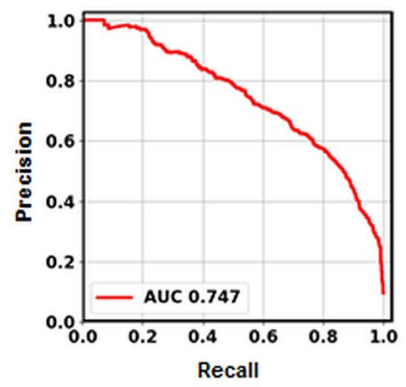

e

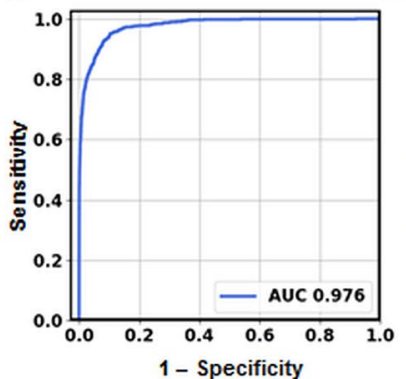

Necrosis

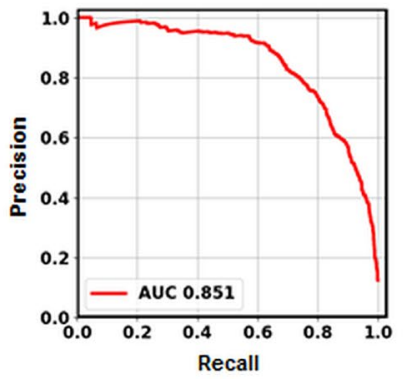

f

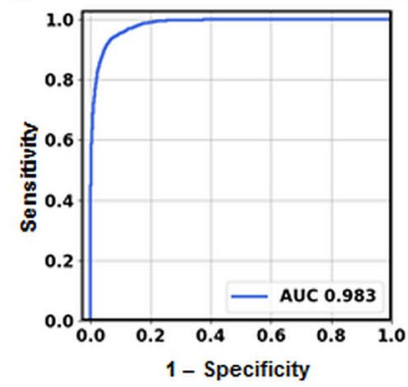

Hemorrhage

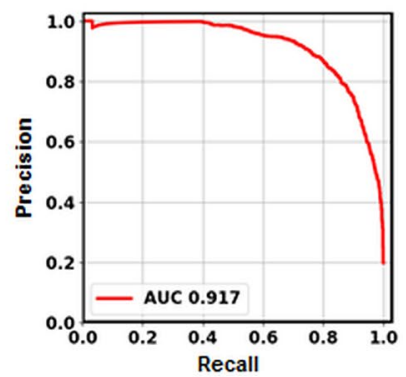

Figure 6. Receiver operating characteristics (ROC) curves and precision-recall (PR) curves calculated using one-vs-rest strategy for 6 different tumor histological components including (a) normal white matter, (b) densely cellular tumor, (c) less densely cellular tumor, (d) tumor infiltrative edge, (e) tumor necrosis and (f) hemorrhage. All 6 ROC curves showed high areas under curve (AUC), indicating strong sensitivity and specificity in detecting these tumor histologic components. Tumor infiltrative edge did not perform as well as other histologic components in precision-recall analysis, indicating that tumor infiltration could be overestimated by the model. Normal WM normal white matter, DC tumor densely cellular tumor, LDC tumor less densely cellular tumor.

\begin{tabular}{|l|l|l|l|l|}
\hline Tumor histology & Sensitivity (\%) & Specificity (\%) & AUC (95\% CI) & F $_{1}$-score \\
\hline Normal WM & $94.1(92.8-97.4)$ & $93.3(89.6-94.3)$ & $0.984(0.982-0.986)$ & 0.918 \\
\hline DC tumor & $88.9(87.2-90.6)$ & $89.8(88.2-91.3)$ & $0.960(0.956-0.963)$ & 0.850 \\
\hline LDC tumor & $97.3(96.3-98.1)$ & $93.8(92.9-94.7)$ & $0.991(0.990-0.993)$ & 0.911 \\
\hline Infiltrative edge & $90.8(87.6-94.2)$ & $86.7(83.2-89.1)$ & $0.950(0.944-0.956)$ & 0.698 \\
\hline Necrosis & $94.5(92.4-96.8)$ & $90.0(87.7-91.6)$ & $0.977(0.973-0.981)$ & 0.799 \\
\hline Hemorrhage & $91.5(88.8-94.4)$ & $92.2(89.2-94.5)$ & $0.976(0.972-0.979)$ & 0.848 \\
\hline
\end{tabular}

Table 2. Diagnostic performances of DHI in classifying different tumor histologies. The $95 \%$ confidence interval (CI) values were calculated using percentile bootstrap method with 10,000 independent experiments. CI confidence interval, Normal WM normal white matter, DC tumor densely cellular tumor, LDC tumor less densely cellular tumor.

the prediction of infiltrative edge was relatively low for AUC (0.747) and in $\mathrm{F}_{1}$-score (0.698), likely due to the highly variable degrees of infiltration or inherent cellularity differences among brain tumors and infiltrated brain regions. For example, infiltrative edges with mild to intermediate tumor cellularity could be falsely predicted to be normal WM. Similar phenomenon was observed from the results of confusion matrix (Fig. 5b). 
In contrast to previous studies, we adopted a voxel-wise analysis through precise co-registration between histology and MR images, to bridge MRI and histology. Application of this approach accurately detected distinct regions within pediatric brain tumors that were histologically heterogeneous ${ }^{37,38}$. Image voxels within a region of interest from a specimen could vary differently from each other, reflecting histological heterogeneity. Since DBSI models diffusion-weighted MRI signals independent of neighboring image voxels ${ }^{10}$, each image voxel has its own DBSI metric profiles. Thus, DBSI provides a unique opportunity to assess the heterogeneous tumor pathology-associated structural changes within individual image voxels. DBSI-derived structural metrics are thus ideal to serve as the unique input classifiers for the DNN algorithm. Patient-wise analysis has been typically studied by correlating image metrics with clinical scores or survival rates. There have been attempts to correlate MRI signal with tumor histopathology using stereotactic biopsy ${ }^{39,40}$. However, the analyses have been hindered by spatial misalignment between MRI-defined lesions and biopsy location, in addition to the high histological heterogeneity of high-grade pediatric brain tumors.

Although the relatively small number of subjects $(n=9)$ limited the broad applicability of the results, we performed voxel-wise analyses (matching the DBSI voxel-based modeling characteristics) on a total of 99,388 image voxels from 45 brain specimens covering different areas of the brain alleviating the limitation on sample size while providing a proof-of-concept demonstration of DHI. The unbalanced data distribution amongst different tumor histologic components imposed another limit since the imbalance could compromise the performance of a DNN model. We have addressed the concern by employing an oversampling approach to balance the training data and adopted precision-recall analyses to provide complement ROC analyses.

In conclusion, we have demonstrated that DHI can accurately characterize and classify multiple histologies in fixed postmortem specimens of pediatric high-grade brain tumors. While precise prediction of infiltrative edges was suboptimal, the collective findings are encouraging. The efficacy of DHI classification of pediatric brain tumor pathologies still requires in vivo application with image-guided stereotactic biopsy validation.

\section{Data availability}

The datasets generated during and/or analyzed during the current study are not publicly available due to sensitive patient information but are available from the corresponding author on reasonable request.

Received: 23 July 2020; Accepted: 8 February 2021

Published online: 26 February 2021

\section{References}

1. Kline, N. E. \& Sevier, N. Solid tumors in children. J. Pediatr. Nurs. 18, 96-102. https://doi.org/10.1053/jpdn.2003.12 (2003).

2. Curtin, S. C., Minino, A. M. \& Anderson, R. N. Declines in cancer death rates among children and adolescents in the United States, 1999-2014. NCHS Data Brief 1-8 (2016).

3. Ostrom, Q. T. et al. CBTRUS statistical report: primary brain and other central nervous system tumors diagnosed in the United States in 2012-2016. Neuro-oncology 21, v1-v100. https://doi.org/10.1093/neuonc/noz150 (2019).

4. Nejat, F., El Khashab, M. \& Rutka, J. T. Initial management of childhood brain tumors: neurosurgical considerations. J. Child Neurol. 23, 1136-1148. https://doi.org/10.1177/0883073808321768 (2008).

5. AlRayahi, J. et al. Pediatric brain tumor genetics: what radiologists need to know. Radiographics 38, 2102-2122. https://doi. org/10.1148/rg.2018180109 (2018).

6. Goo, H. W. \& Ra, Y. S. Advanced MRI for pediatric brain tumors with emphasis on clinical benefits. Korean J. Radiol. 18, $194-207$. https://doi.org/10.3348/kjr.2017.18.1.194 (2017).

7. Panigrahy, A. \& Bluml, S. Neuroimaging of pediatric brain tumors: from basic to advanced magnetic resonance imaging (MRI). J. Child Neurol. 24, 1343-1365. https://doi.org/10.1177/0883073809342129 (2009).

8. Plaza, M. J., Borja, M. J., Altman, N. \& Saigal, G. Conventional and advanced MRI features of pediatric intracranial tumors: posterior fossa and suprasellar tumors. AJR Am. J. Roentgenol. 200, 1115-1124. https://doi.org/10.2214/AJR.12.9725 (2013).

9. Reardon, D. A. \& Wen, P. Y. Glioma in 2014: unravelling tumour heterogeneity-implications for therapy. Nat. Rev. Clin. Oncol. 12, 69-70. https://doi.org/10.1038/nrclinonc.2014.223 (2015).

10. Wang, Y. et al. Quantification of increased cellularity during inflammatory demyelination. Brain 134, 3590-3601. https://doi. org/10.1093/brain/awr307 (2011).

11. Wang, Y. et al. Differentiation and quantification of inflammation, demyelination and axon injury or loss in multiple sclerosis. Brain 138, 1223-1238. https://doi.org/10.1093/brain/awv046 (2015).

12. Sun, P. et al. Diffusion basis spectrum imaging provides insights into MS pathology. Neurol. Neuroimmunol. Neuroinflamm. 7 , e655. https://doi.org/10.1212/nxi.0000000000000655 (2020).

13. Ye, Z. et al. Deep learning with diffusion basis spectrum imaging for classification of multiple sclerosis lesions. Ann. Clin. Transl. Neurol. 7, 695-706. https://doi.org/10.1002/acn3.51037 (2020).

14. Ye, Z. et al. The impact of edema and fiber crossing on diffusion MRI metrics assessed in an ex vivo nerve phantom: multi-tensor model vs. diffusion orientation distribution function. NMR Biomed. https://doi.org/10.1002/nbm.4414 (2021).

15. Sun, P. et al. Diffusion assessment of cortical changes, induced by traumatic spinal cord injury. Brain Sci. https://doi.org/10.3390/ brainsci7020021 (2017).

16. Zhan, J. et al. Diffusion basis spectrum and diffusion tensor imaging detect hippocampal inflammation and dendritic injury in a virus-induced mouse model of epilepsy. Front. Neurosci. Switz. https://doi.org/10.3389/fnins.2018.00077 (2018).

17. Ye, Z. et al. Diffusion histology imaging combining diffusion basis spectrum imaging (DBSI) and machine learning improves detection and classification of glioblastoma pathology. Clin. Cancer Res. https://doi.org/10.1158/1078-0432.CCR-20-0736 (2020).

18. Chawla, N. V., Bowyer, K. W., Hall, L. O. \& Kegelmeyer, W. P. SMOTE: Synthetic minority over-sampling technique. J. Artif. Intell. Res. 16, 321-357. https://doi.org/10.1613/jair.953 (2002).

19. Haibo, H., Yang, B., Garcia, E. A. \& Shutao, L. 2008 IEEE International Joint Conference on Neural Networks (IEEE World Congress on Computational Intelligence) 1322-1328.

20. Mart et al. Proceedings of the 12th USENIX Conference on Operating Systems Design and Implementation 265-283 (USENIX Association, Savannah, GA, USA, 2016).

21. Terrier, L.-M. et al. Natural course and prognosis of anaplastic gangliogliomas: a multicenter retrospective study of 43 cases from the French Brain Tumor Database. Neuro-Oncology 19, 678-688. https://doi.org/10.1093/neuonc/now186 (2017).

22. Ruopp, M. D., Perkins, N. J., Whitcomb, B. W. \& Schisterman, E. F. Youden Index and optimal cut-point estimated from observations affected by a lower limit of detection. Biom. J. 50, 419-430. https://doi.org/10.1002/bimj.200710415 (2008). 
23. Carpenter, J. \& Bithell, J. Bootstrap confidence intervals: when, which, what? A practical guide for medical statisticians. Stat. Med. 19, 1141-1164. https://doi.org/10.1002/(Sici)1097-0258(20000515)19:9\%3c1141::Aid-Sim479\%3e3.0.Co;2-F (2000).

24. Virtanen, P. et al. SciPy 1.0: fundamental algorithms for scientific computing in Python. Nat. Methods 17, 261-272. https://doi. org/10.1038/s41592-019-0686-2 (2020).

25. Pedregosa, F. et al. Scikit-learn: machine learning in python. J. Mach. Learn. Res. 12, 2825-2830 (2011).

26. Vanan, M. I. \& Eisenstat, D. D. Management of high-grade gliomas in the pediatric patient: past, present, and future. Neuro-oncology Pract. 1, 145-157. https://doi.org/10.1093/nop/npu022 (2014).

27. Pfister, S., Hartmann, C. \& Korshunov, A. Histology and molecular pathology of pediatric brain tumors. J. Child Neurol. 24, 1375-1386. https://doi.org/10.1177/0883073809339213 (2009).

28. McGirt, M. J., Villavicencio, A. T., Bulsara, K. R. \& Friedman, A. H. MRI-guided stereotactic biopsy in the diagnosis of glioma: comparison of biopsy and surgical resection specimen. Surg. Neurol. 59, 277-281 (2003) (discussion 281-272).

29. Apuzzo, M. L., Chandrasoma, P. T., Cohen, D., Zee, C. S. \& Zelman, V. Computed imaging stereotaxy: experience and perspective related to 500 procedures applied to brain masses. Neurosurgery 20, 930-937 (1987).

30. Air, E. L., Warnick, R. E. \& McPherson, C. M. Management strategies after nondiagnostic results with frameless stereotactic needle biopsy: retrospective review of 28 patients. Surg. Neurol. Int. 3, S315-319. https://doi.org/10.4103/2152-7806.103026 (2012).

31. Mabray, M. C., Barajas, R. F. Jr. \& Cha, S. Modern brain tumor imaging. Brain Tumor Res. Treat. 3, 8-23. https://doi.org/10.14791 /btrt.2015.3.1.8(2015).

32. Villanueva-Meyer, J. E., Mabray, M. C. \& Cha, S. Current clinical brain tumor imaging. Neurosurgery 81, 397-415. https://doi. org/10.1093/neuros/nyx103 (2017).

33. Kumar, A. J. et al. Malignant gliomas: MR imaging spectrum of radiation therapy- and chemotherapy-induced necrosis of the brain after treatment. Radiology 217, 377-384. https://doi.org/10.1148/radiology.217.2.r00nv36377 (2000).

34. Price, S. J. et al. Diffusion tensor imaging of brain tumours at 3T: a potential tool for assessing white matter tract invasion?. Clin. Radiol. 58, 455-462. https://doi.org/10.1016/s0009-9260(03)00115-6 (2003).

35. Scherer, H. J. Structural development in gliomas. Am. J. Cancer 34, 333-351. https://doi.org/10.1158/ajc.1938.333 (1938).

36. Kono, K. et al. The role of diffusion-weighted imaging in patients with brain tumors. AJNR Am. J. Neuroradiol. 22, 1081-1088 (2001).

37. Gajjar, A., Pfister, S. M., Taylor, M. D. \& Gilbertson, R. J. Molecular insights into pediatric brain tumors have the potential to transform therapy. Clin. Cancer Res. 20, 5630-5640. https://doi.org/10.1158/1078-0432.CCR-14-0833 (2014).

38. Pollack, I. F., Agnihotri, S. \& Broniscer, A. Childhood brain tumors: current management, biological insights, and future directions. J. Neurosurg. Pediatr. 23, 261. https://doi.org/10.3171/2018.10.Peds18377 (2019).

39. Gauvain, K. M. et al. Evaluating pediatric brain tumor cellularity with diffusion-tensor imaging. Am. J. Roentgenol. 177, 449-454. https://doi.org/10.2214/ajr.177.2.1770449 (2001).

40. Eidel, O. et al. Tumor infiltration in enhancing and non-enhancing parts of glioblastoma: a correlation with histopathology. PLoS ONE 12, e0169292. https://doi.org/10.1371/journal.pone.0169292 (2017).

\title{
Acknowledgements
}

This work was supported by The Taylor Rozier Hope for a Cure Foundation (J.B.R.), The Josie Foundation (J.B.R.), Matt's Hats Foundation (J.B.R.), The Derek Griffitts Foundation (J.B.R.), and The Kwesi Prince Foundation (J.B.R.), and in part by NIH R01-NS047592 (S.K.S.), P01-NS059560 (S.K.S.), and U01-EY025500 (S.K.S.). The authors are indebted to the patients and their families for donating the brains without which this research would not have been possible.

\section{Author contributions}

Z.Y., K.S. and J.L. wrote the manuscript. Z.Y., K.S. and J.V. performed the experiments. Z.Y., J.L., P.S., and C.S. analyzed the experimental data. S.D. and K.S. performed the histopathologic evaluations. Z.Y. and A.T.W. developed the classifier and conducted the statistical analyses. J.B.R., S.D. and A.M. provided surgical specimens for imaging. S.D., J.B.R., and S.K.S. interpreted data, designed and supervised the research, and supervised the writing of the manuscript. All authors have reviewed and approved the final version of the manuscript.

\section{Competing interests}

S.K.S. has a financial [ownership] interest in CancerVision LLC and may financially benefit if the company is successful in marketing its product(s) that is/are related to this research. Other authors declared no competing interests.

\section{Additional information}

Correspondence and requests for materials should be addressed to S.-K.S., S.D. or J.B.R.

Reprints and permissions information is available at www.nature.com/reprints.

Publisher's note Springer Nature remains neutral with regard to jurisdictional claims in published maps and institutional affiliations.

\begin{abstract}
Open Access This article is licensed under a Creative Commons Attribution 4.0 International License, which permits use, sharing, adaptation, distribution and reproduction in any medium or format, as long as you give appropriate credit to the original author(s) and the source, provide a link to the Creative Commons licence, and indicate if changes were made. The images or other third party material in this article are included in the article's Creative Commons licence, unless indicated otherwise in a credit line to the material. If material is not included in the article's Creative Commons licence and your intended use is not permitted by statutory regulation or exceeds the permitted use, you will need to obtain permission directly from the copyright holder. To view a copy of this licence, visit http://creativecommons.org/licenses/by/4.0/.
\end{abstract}

(C) The Author(s) 2021 